\title{
Rénovation et réhabilitation des grands ensembles dans les nouveaux Länder : quel avenir pour la ville socialiste?
}

Restructuring and rehabilitation in new Länder: what does the future hold for the socialist town?

Stadtumbau und Sanierung der Großwohnsiedlungen in den neuen Ländern : welche Zukunft für die sozialistische Stadt?

\section{Axelle De Gaspérin}

\section{OpenEdition}

\section{Journals}

Édition électronique

URL : https://journals.openedition.org/rge/1469

DOI : $10.4000 /$ rge. 1469

ISSN : 2108-6478

Éditeur

Association des géographes de l'Est

Édition imprimée

Date de publication : 1 juin 2006

ISSN : 0035-3213

\section{Référence électronique}

Axelle De Gaspérin, «Rénovation et réhabilitation des grands ensembles dans les nouveaux Länder : quel avenir pour la ville socialiste ? », Revue Géographique de l'Est [En ligne], vol. 46 / 3-4 | 2006, mis en ligne le 18 décembre 2009, consulté le 23 novembre 2021. URL : http://journals.openedition.org/rge/ 1469 ; DOI : https://doi.org/10.4000/rge.1469

Ce document a été généré automatiquement le 23 novembre 2021.

Tous droits réservés 


\title{
Rénovation et réhabilitation des grands ensembles dans les nouveaux Länder : quel avenir pour la ville socialiste?
}

\author{
Restructuring and rehabilitation in new Länder : what does the future hold for \\ the socialist town? \\ Stadtumbau und Sanierung der Großwohnsiedlungen in den neuen Ländern : \\ welche Zukunft für die sozialistische Stadt?
}

Axelle De Gaspérin

\section{Introduction}

1 Si la question du devenir des grands ensembles est un sujet de préoccupation particulièrement important et déjà relativement ancien pour les villes d'Europe occidentale, elle est devenue aussi un problème majeur pour les villes d'Europe centrale et orientale marquées par quarante ans de régime socialiste. En effet, celui-ci a légué un très important parc immobilier constitué de grands ensembles qui pouvaient rassembler jusqu'à plus de la moitié de la population totale des villes. Or, ces logements qui se voulaient à l'image d'un modèle idéologique qui s'est effondré en 1989, ont vieilli, se sont dégradés et sont plus ou moins frappés de vétusté, nécessitant d'importants travaux de réaménagement afin d'adapter ce cadre urbain hérité.

Dans les nouveaux Länder, ce problème se pose avec une singulière acuité parce qu'en raison du contexte particulier de la réunification allemande, les villes de l'ex-RDA (République Démocratique Allemande) ont connu une évolution originale, marquée par un déclin démographique d'une ampleur exceptionnelle et par une puissante périurbanisation. Aussi, toutes les villes est-allemandes et en particulier les quartiers de grands ensembles ont vu leur population très fortement diminuer, ce qui s'est 
traduit par une rapide augmentation du nombre de logements vacants nécessitant une profonde restructuration. Il s'agit à la fois d'adapter le cadre urbain à une population ayant fortement diminué et de rendre une certaine attractivité à des quartiers à l'image souvent dégradée et dont il faut éviter qu'ils deviennent des espaces de concentration des populations les plus pauvres. Les autorités municipales en charge de ces grands ensembles sont donc confrontées à un défi de taille pour lequel elles ont reçu l'aide de l'État fédéral à l'origine d'un programme de réhabilitation et de rénovation des villes appelé Stadtumbau Ost. A partir des exemples de deux villes des nouveaux Länder, Eisenhüttenstadt et Halle, on présentera ici les principaux aspects de ce programme qui, par son ampleur, est sans équivalent en Europe. La situation urbaine des nouveaux Länder et les programmes d'urbanisme mis en œuvre méritent un intérêt tout particulier dans une réflexion générale sur l'urbain et l'urbanisme dans les pays d'Europe occidentale. Les difficultés rencontrées par les villes y sont en effet d'une rare gravité.

\section{Les villes des nouveaux länder : de la ville compacte à la ville qui se rétracte}

\section{A. Des paysages urbains originaux}

3 Le processus urbain de l'ex-RDA a suivi un cours particulier, dont l'analyse se révèle riche d'enseignements pour une étude générale du fait urbain. Dans le cadre de cette contribution, un retour en arrière nous permettra d'appréhender plus aisément leur situation actuelle. Nous ne reviendrons pas ici sur leur apparition et leur développement, qui se rattachent à l'histoire générale des villes européennes; mais une rupture majeure a lieu avec le passage sous influence soviétique au lendemain de la Seconde Guerre mondiale.

4 La ville a été un champ d'étude privilégié dans l'histoire de l'URSS. L'objectif affirmé était de trouver une forme urbaine qui exprimerait la société socialiste ; cependant, les heurts et revirements au gré des ruptures politiques, idéologiques et économiques ont eu des répercussions tant sur la manière d'appréhender l'urbain que sur la concrétisation de celui-ci. On peut grossièrement distinguer une première période, entre l'instauration du nouveau régime et l'extrême fin des années 1920, une seconde qui correspond au cœur des années de régime stalinien, une troisième qui s'étend de la remise en cause de l'ère stalinienne (en décembre 1954, Khrouchtchev convoque les architectes d'URSS et les invite à développer des programmes moins coûteux) à la fin des années 1980. La première période est caractérisée par un intense bouillonnement intellectuel dans tous les domaines, avec la volonté d'inventer de nouvelles conditions d'existence pour un homme nouveau ; le cadre bâti est concerné au premier chef. Le cadre de vie était en effet envisagé comme un véritable « appareil orthopédique (Kopp, 1972) pour corriger et transformer l'homme. Les solutions de collectivisme les plus poussées sont proposées ; pour certains, il faut abolir la distinction ville-campagne. On propose par exemple d'implanter le long des axes de communication des cellules individuelles (Kopp, 1978). Entre 1929 et 1931, un coup d'arrêt brutal est mis à ce foisonnement, avec la volonté de Staline de revenir à une conception traditionnelle de la ville: la densité est à nouveau valorisée, et l'on cherche à réintroduire dans l'architecture des éléments appartenant à la culture nationale. Ce revirement 
s'explique entre autres par le désir d'établir un contre-modèle aux recherches menées dans les pays occidentaux et dont on juge les recherches de l'avant-garde trop proches. L'extension de l'influence soviétique aux pays d'Europe de l'Est après la Seconde Guerre Mondiale entraîne l'application des principes d'urbanisme définis à Moscou dans ses satellites : les territoires de l'Est de l'Allemagne connaîtront brièvement cette période de « construction dans la tradition nationale » (nationale Bautradition), période dont est issue le cœur d'Eisenhüttenstadt. Cette période se traduit par une architecture monumentale, une voirie surdimensionnée qui concourent, en dépit des allégations nationales, à un paysage urbain assez semblable à celui de nombreuses dictatures. Comme le souligne Jay Rowell pour le cas de la RDA, «la crise du logement n'était pas pensée en termes quantitatifs, mais plutôt en termes symboliques - il ne s'agissait nullement de construire des logements en masse (...) mais plutôt de marquer symboliquement le territoire avec des "palais» pour les ouvriers construits en centre-ville, sacrifiant la quantité au nom de la qualité» (Rowell, 2004).

5 La mort de Staline entraine un changement radical avec l'orientation vers des solutions plus rapides et moins coûteuses, en raison notamment des retards énormes pris dans la construction des logements. On se tourne vers la construction industrialisée développée dans les pays occidentaux. Tout au long de la première moitié du $\mathrm{XX}^{\mathrm{e}}$ siècle, en effet, a été forgée la ville moderne, et les réflexions proposées par différents groupes et courants (Bauhaus, Congrès internationaux d'architecture moderne...) ont conduit à privilégier l'industrialisation de la construction. La concrétisation de la ville moderne se fera essentiellement, tant à l'Est qu'à l'Ouest, par le biais des grands ensembles. Cette troisième période va couvrir l'URSS et ses satellites de grands ensembles, correspondant certes à une vision moderne de la vie collective, mais surtout à la convergence de divers impératifs. "En principe, la construction industrialisée offre les meilleures performances pour égaliser les conditions de logement - et donc participer à l'édification d'une société sans classes» (Coudroy de Lille, 2004). En fait, la construction industrialisée ouvre la possibilité d'une gestion de la production de logements au niveau étatique, qui peut alors se calquer sur les besoins en main-d'oeuvre du secteur industriel. Ainsi, la construction de logements va être privilégiée autour des complexes industriels. Enfin, la construction industrialisée permet de réduire les coûts de construction. L'urbain continue de faire l'objet de réflexions; en témoigne, comme nous le verrons par la suite, la construction de Halle-Neustadt en RDA; mais les difficultés économiques, les retards accumulés rendent finalement illusoire la continuité entre le plan des recherches et celui de la construction des villes ou quartiers.

6 Ainsi, de la fin des années cinquante à 1989, les villes de RDA vont, dans leur grande majorité, voir pousser ces grands ensembles; par leur emprise spatiale, souvent démesurée, et leur implantation généralisée, ils sont une caractéristique du paysage urbain de ce pays disparu. Un colloque sur le thème «Les grands ensembles dans les villes européennes » s'est tenu en 1996 à Leipzig et a donné lieu à la publication de plusieurs articles, qui fournissent un apport incontournable sur ces formes de développement urbain définies comme étant des « ensembles d'habitation comptant plus de 2500 logements, construits après la Seconde Guerre mondiale en suivant un concept urbanistique uniforme, avec des bâtiments comptant plusieurs étages" (Müller, 1997). Quelques différences méritent cependant d'être soulignées entre les grands ensembles de l'ancienne RDA et ceux des pays d'Europe de l'Ouest. 
7 On a souligné les raisons essentielles qui ont conduit en RDA à privilégier ce type de quartier. La multiplication des grands ensembles s'y est accompagnée d'une absence d'entretien des quartiers anciens des centres-villes, ainsi que des extensions du $\mathrm{XIX}^{\mathrm{e}}$ siècle et de la première moitié du $\mathrm{XX}^{\mathrm{e}}$ siècle durant de longues années. Ils présentent encore aujourd'hui pour certains un aspect dégradé, en dépit des mesures déjà mises en œuvre. Les efforts de construction ayant porté, au cours des années socialistes, presque exclusivement sur les grands ensembles en périphérie des villes, il en résulte un développement spatial des villes relativement limité qui s'oppose à «l'urban sprawl» caractéristique des villes américaines. Les villes des pays socialistes sont des villes « compactes ».

L'accent mis sur ce type d'urbanisme en explique la prégnance encore aujourd'hui dans les anciens pays socialistes, où environ un tiers de la population vit dans de tels quartiers. Dans les grandes villes, la proportion est d'ailleurs souvent beaucoup plus grande puisque entre 36 et $40 \%$ des habitants de Varsovie, de Prague et de Budapest habitent dans des grands ensembles et cette proportion s'élève même à $77 \%$ à Bratislava et à $82 \%$ à Bucarest (Deshaies, 2005). A l'échelle de l'Allemagne, on est frappé par le contraste existant entre anciens et nouveaux Länder en ce qui concerne l'importance des grands ensembles : alors qu'en 1994, 23 \% de la population de l'ex-RDA vivait dans un grand ensemble, c'était seulement $1,7 \%$ pour la RFA qui, parmi les pays d'Europe occidentale est l'un de ceux qui en compte le moins. Au total, près de 3,1 millions de logements ont été construits en RDA entre 1950 et 1990, dont 2,7 millions après 1960 , soit $38 \%$ du nombre total de logements existant en 1990 . Sur ces 2,7 millions, $48 \%$ étaient situés dans des grands ensembles comptant plus de 2500 logements (fig. 1). Les plus grands d'entre eux, à Berlin-Henningsdorf, Halle-Neustadt et Leipzig-Grünau dépassent 30000 logements et Berlin-Marzahn atteint même 59200 logements pour 167000 habitants (Holz, 1997, fig. 1). C'est ainsi que dans les grandes villes comme Leipzig, Dresde ou Halle, près d'un tiers de la population habite dans des grands ensembles, mais dans les villes nouvelles créées autour d'un complexe industriel comme Eisenhüttenstadt, Schwedt ou Hoyerswerda c'est plus des trois quarts de la population. 
Figure 1 : Les grands ensembles en RDA

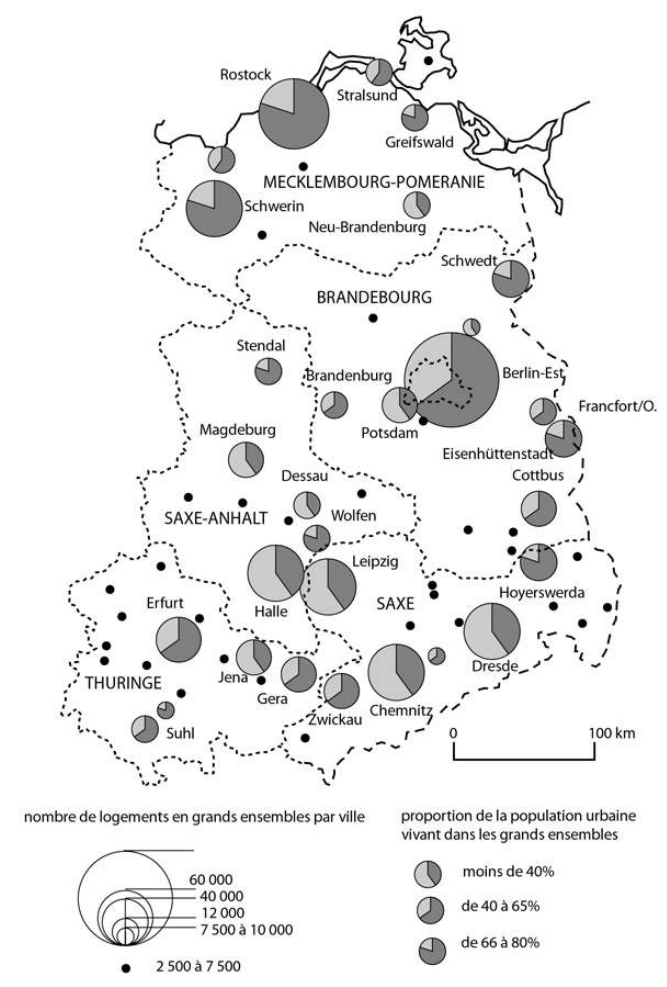

9 Par ailleurs, il faut souligner que les grands ensembles de l'Est constituent des zones d'une uniformité beaucoup plus grande que ceux existant en Europe occidentale. Qui plus est, nombre d'équipements prévus n'ont pas été réalisés : espaces verts, terrains de jeux, transports en commun par exemple. Les raisons de cette moindre qualité, qui concerne aussi souvent le bâti lui-même, sont à rechercher dans les restrictions économiques. Ces quartiers sont appelés en ex-RDA Plattenbausiedlung, terme forgé à partir de Plattenbau, vocable qui désigne les plaques préfabriquées, prévues avec portes et fenêtres, éléments de base qu'il suffisait d'assembler sur le chantier.

Ce bref retour en arrière nous a permis de peindre rapidement le contexte dans lequel sont apparus les grands ensembles en RDA. La construction successive de quatre villes nouvelles permet de saisir à quel point l'urbanisme avait pour le régime un rôle de vitrine. Eisenhüttenstadt fut la première (1951) suivi de Hoyerswerda (1955), Schwedt/ Oder (1961) et enfin Halle-Neustadt (1963). Cependant, si les grands ensembles, et de manière plus générale le mouvement moderne, ont répondu à un moment donné à l'idéologie en vigueur en URSS puis dans ses satellites, les changements brusques en matière de pensée urbanistique montrent bien l'allégeance à d'autres contraintes, politiques et économiques. Ainsi, on pourrait parler d'une conjonction « $a$ posteriori »(Coudroy de Lille, 2004) entre l'idéologie socialiste et les grands ensembles.

\section{B. Un déclin démographique inquiétant}

11 Les phénomènes qui touchent les nouveaux Länder d'un point de vue économique et démographique fragilisent leurs structures et placent le devenir de ces régions sous le sceau de l'incertitude. Dès avant la réunification, les départs vers la RFA et la faible natalité rendaient la situation démographique inquiétante, et cela n'a fait que 
s'accentuer au lendemain de la réunification. De 1989 à 2005 les cinq Länder orientaux (Berlin non compris) ont ainsi perdu 1,7 million d'habitants, soit $11 \%$ de leur population. Si la diminution a été particulièrement rapide au début des années quatrevingt dix, elle se poursuit encore et c'est ainsi qu'entre 1995 et 2005, la population a diminué d'environ 700000 habitants, soit $5 \%$. En outre, l'une des conséquences de cette évolution est le vieillissement de la population. D'un point de vue économique, certains spécialistes n'hésitent pas à parler de désindustrialistion et de dé-économisation (DeÖkonomisierung) (Hannemann, 2003), avec la fermeture de très nombreux sites industriels, qui constituaient l'armature de l'économie est-allemande. A l'heure actuelle, le taux de chômage est l'un des indicateurs d'une situation économique toujours peu reluisante (il était de $19,4 \%$, contre $10,2 \%$ dans les anciens Länder en avril 2006) ${ }^{1}$.

Les villes ont été particulièrement touchées par ce déclin démographique, entrânant un délabrement de certains quartiers et une augmentation des coûts pour les habitants restés sur place: le maintien d'un équilibre économique apparaît de plus en plus difficile. Deux raisons majeures à ce phénomène: le déclin démographique général, avec de nombreux départs vers l'Ouest et une natalité très basse d'une part, un mouvement des villes vers leur périphérie, avec une volonté d'accession à la propriété d'autre part. La plupart des grandes villes ou des villes moyennes ont perdu entre 10 et $20 \%$ de leur population, du moins avant que la réforme administrative et notamment les regroupements de communes ne leur permettent de « récupérer » en quelque sorte une partie des habitants qui s'étaient diffusés dans l'espace périurbain. De 1989 à 1997, Leipzig a perdu près de 100000 habitants, passant d'une population de 545000 à 450000 habitants. Des villes de plus de 300000 habitants, comme Chemnitz ou Halle, ont connu une diminution de plus de 50000 habitants, soit un sixième de leur population. Mais alors que grâce à la réforme communale les deux plus grandes villes d'Allemagne de l'Est, Dresde et Leipzig, ont chacune regagné plusieurs dizaines de milliers d'habitants et ont désormais une évolution pratiquement stabilisée, toutes les autres villes continuent à perdre de la population à un rythme parfois rapide. C'est ainsi que depuis 1998 Chemnitz et Halle ont encore perdu chacune près de 25000 habitants, soit $9 \%$ de leur population. Les villes monoindustrielles créées au temps de la RDA comme Eisenhüttenstadt, Schwedt ou Hoyerswerda ont subi des pertes encore plus massives qui dépassent un tiers de leur population de 1989 et comme leur déclin se poursuit à un rythme rapide, c'est même le devenir de ces villes à l'horizon 2020-2030 qui est particulièrement inquiétant.

13 Ces départs nombreux se répercutent sur les logements et entraînent le développement d'un inquiétant phénomène d'inoccupation: en 2002, 1,03 millions de logements étaient vides dans l'ensemble des nouveaux Länder, soit 14,7\%. Selon une étude de la commission «Mutation structurelle du secteur du logement dans les nouveaux Länder $»^{2}$ en 1990, 400000 logements étaient insalubres. Le nombre de logements vides a ensuite augmenté par suite des constructions nouvelles, en dépit d'un nombre de ménages croissant (plus 600 000). Certes, il faut souligner qu'une part importante des logements vides est située dans des immeubles anciens de centre-ville (un tiers des logements construits avant 1918 sont inoccupés). Cependant, dans les immeubles édifiés entre 1949 et 1990, le taux d'inoccupation qui était encore relativement faible en 1998, a fortement augmenté depuis, et devient donc également un problème majeur. 
14 À Leipzig par exemple, la proportion de logements vides dans le parc immobilier construit entre 1949 et 1990 a progressé de $67 \%$ entre mars 2000 et décembre 2002 pour atteindre environ $15 \%$, soit plus de 15000 logements (Schmidt et al. 2005). Dans les villes monoindustrielles, particulièrement frappées par les restructurations consécutives à la réunification, la situation est beaucoup plus préoccupante. Ainsi, Hoyerswerda, la principale ville du bassin de lignite de Basse Lusace, a perdu $36 \%$ de sa population depuis 1990 et ne comptait plus que 43000 habitants à la fin de l'année 2005. Or, il faut souligner qu'à Hoyerswerda, c'est dans les quartiers de grands ensembles construits depuis les années 1960 que la diminution de population a été proportionnellement la plus importante. Aussi, dans cette ville, ce sont plus de 4500 logements qui étaient inoccupés en 2003 et ce, malgré la démolition de plus de 1500 logements. Or, la poursuite du déclin démographique de la ville pourrait réduire la population à 35000 habitants en 2015, voire même en 2010 suivant les prévisions les plus pessimistes. Dans ces conditions, c'est donc 5000 à 6000 logements supplémentaires qui pourraient devenir vacants et il est ainsi prévu de démolir environ 8500 logements, soit un tiers du parc immobilier existant.

Tableau 1 : Évolution de la population et logements vides dans quelques villes nouvelles et grands ensembles dans les nouveaux Länder

\begin{tabular}{|c|c|c|c|c|c|}
\hline Ville & Nbre hab. 1992 & Nbre hab. & $\begin{array}{c}\text { de diminution } \\
\text { de la pop. }\end{array}$ & Nbre logements & $\begin{array}{l}\% \text { logements } \\
\text { vides }\end{array}$ \\
\hline Berlin Hellersdorf & 108700 & $86300(1999)$ & $-21 \%$ & 42000 & $9 \%$ (1999) \\
\hline Jena Lobeda & 32000 & 22375 (2001) & $-30 \%$ & 12970 & $12 \%(1999)$ \\
\hline Rostock Groß Klein & 22250 & $11800(2002)$ & $-47 \%$ & - & - \\
\hline Rostock Schmarl & 13800 & $8700(2002)$ & $-37 \%$ & 5075 & $11 \%(2000)$ \\
\hline Halle Neustadt & 84672 & $54000(2004)$ & $-36 \%$ & 35600 & - \\
\hline Halle Silberhöhe & 37800 & $19000(2004)$ & $-50 \%$ & 14700 & - \\
\hline Schwerin Mueßer Holz & 26400 (1995) & $14400(2003)$ & $-45 \%$ & - & - \\
\hline Schwedt & 50516 & $37445(2004)$ & $-26 \%$ & 21044 & $\begin{array}{c}24,5 \% \text { ( } 2000, \\
\text { avant dêmolition) }\end{array}$ \\
\hline $\begin{array}{l}\text { Hoyerswerda (avant } \\
\text { absorption de communes) }\end{array}$ & $65685(1990)$ & 40811 (2002) & $-38 \%$ & 27418 & $\begin{array}{l}22 \%(2000, \\
\text { avant démolition) }\end{array}$ \\
\hline
\end{tabular}

Le déclin de la population met les villes dans une situation particulièrement délicate. Le grand nombre de logements vides est à la fois une conséquence spectaculaire et un indicateur du déclin de la population. Il engendre une mutiplicité de phénomènes inquiétants pour les communes: la sous-utilisation des équipements collectifs, une augmentation des coûts (ramassage des ordures, entretien de la voirie, chauffage collectif, alimentation en eau potable...) pour les habitants, les incitant plus encore au départ. Les communes connaissent des difficultés financières, ainsi que les entreprises immobilières. Au sein de la question générale de la rénovation urbaine, la situation des grands ensembles pose certains problèmes particuliers. Bien que les logements vides soient plus nombreux dans le parc immobilier ancien, dans tous les grands ensembles il y a une forte déperdition de population qui s'est accélérée depuis la fin des années quatre-vingt-dix. On note un rejet fréquent de cette forme urbaine, ce qu'illustre parfaitement la situation française. Les raisons en sont très complexes, mais on peut mettre en avant quelques facteurs. La qualité du bâti, et ce particulièrement dans les quartiers les plus récents des grands ensembles des nouveaux Länder, a été sacrifiée aux impératifs économiques. La qualité paysagère, avec la pauvreté et la répétition des formes géométriques, combinée au surdimensionnement ne semble pas favoriser 
l'appropriation du quartier par les habitants. Les grands ensembles ont été conçus comme un produit, en négligeant le fait que l'habitat ne peut se réduire à cet aspect.

\section{Le programme Stadtumbau Ost}

\section{A. Le programme Stadtumbau Ost, une stratégie intégrée de développement urbain}

Le gouvernement fédéral a réagi en lançant le programme Stadtumbau Ost (Rénovation Urbaine à l'est). En France, l'expression "rénovation urbaine » désigne une opération de complet remodelage d'un immeuble ou d'un quartier, passant par une démolition complète ou quasi-complète à laquelle succède une reconstruction. Selon le Dictionnaire de l'urbanisme et de l'aménagement (Merlin, Choay, 2005), la formulation de rénovation urbaine n'est pas adéquate: on devrait lui préférer celle de «démolitionreconstruction", et employer le terme rénovation en lieu et place de celui de réhabilitation. En France, c'est au cours des Trente Glorieuses que la rénovation urbaine a été massivement choisie comme mode de transformation, en particulier des quartiers anciens. Les critiques nombreuses formulées à l'encontre de la rénovation ont rapidement conduit à adopter plutôt la pratique de la réhabilitation, c'est-à-dire le « rétablissement d'un édifice ou d'un ensemble d'immeubles dans ses capacités à abriter des activités et des habitants. Les principales caractéristiques héritées de l'objet traité sont alors préservées et insérées dans un nouveau fonctionnement mieux adapté au temps présent. » (Lévy, Lussault, 2003). Aujourd'hui, l'expression rénovation urbaine a trouvé un second souffle avec la question du devenir des quartiers sensibles dans les banlieues. L'intervention sur le plan urbain prévoit des opérations diverses (résidentialisation, réhabilitation d'immeubles, requalification des espaces verts, ajout ou transformation d'équipements...), regroupées sous l'appellation générale rénovation urbaine, emploi que le Dictionnaire de l'urbanisme et de l'aménagement estime plus en adéquation avec son étymologie.

Il nous a paru opportun de traduire le nom du programme Stadtumbau Ost par "Rénovation Urbaine à l'est », de préférence à d'autres traductions, "Transformation Urbaine à l'est » par exemple. Le terme transformation, quoique traduction appropriée du terme Umbau, ne nous paraît pas à même de rendre compte des exigences contenues dans le programme, pas plus que de son ampleur. Certes l'expression rénovation urbaine est sujette comme on l'a vu à quelques ambiguïtés, mais elle nous a paru recouvrir plusieurs éléments importants de la politique suivie avec le programme Stadtumbau Ost: les idées de démolition et de reconstruction, mais aussi la notion de regroupement de plusieurs types d'opérations, notamment de réhabilitation. Il ne faut cependant pas faire un amalgame entre le programme national de rénovation urbaine porté par l'ANRU (Agence Nationale de Rénovation Urbaine), lancé en France en 2003, et le programme Stadtumbau Ost, en dépit de certaines convergences.

Le programme Stadtumbau Ost a démarré au premier janvier 2002 ; le gouvernement fédéral investit pour une période de huit ans (2002-2009) 1 milliard d'euro environ. Au total, ce sont environ 2,5 milliards d'euro qui doivent être investis par le gouvernement fédéral, les Länder et les communes, mais cette somme s'entend comme prenant en compte les moyens accordés pour favoriser l'accession à la propriété dans les quartiers anciens. Le programme Stadtumbau Ost,en effet, s'il est une partie fondamentale de la 
rénovation urbaine, doit être compris au sein d'un ensemble de mesures, qui visent à assurer l'avenir des villes par l'assainissement du marché immobilier, l'amélioration du cadre bâti, l'adaptation des structures à la nouvelle donne démographique, la revitalisation du centre-ville, la lutte contre la désagrégation et la ségrégation. Pour cela, deux autres mesures sont particulièrement importantes : d'une part favoriser par des aides l'accession à la propriété dans le bâti existant, notamment dans le tissu ancien; d'autre part, étant donnée la forte proportion de logements en location dans les quartiers centraux, aider les investisseurs qui proposent des appartements à la location.

Le programme Stadtumbau Ost se divise entre opérations de démolition et de revalorisation ; il comporte également un concours pour la mise en place des concepts intégrés de développement urbain (integrierte Stadtentwicklungskonzepte). L'aide fédérale se monte à $50 \%$ pour les démolitions, $33 \%$ pour la revalorisation; les coûts restants sont assumés par les communes et les Länder. Il est prévu de démolir entre 2002 et 2009 environ 350000 logements; en octobre 2005, 104000 environ ont d'ores et déjà été détruits dans le cadre du programme, auxquels on peut ajouter 22300 démolitions hors programme. Si les démolitions sont une mesure phare, elles doivent être considérées avant tout comme un moyen, et non comme un but. La consolidation des quartiers dégradés, menacés par les phénomènes de ségrégation, ainsi que la revalorisation et la revitalisation des quartiers anciens, qui participent à l'identité de la ville et sont souvent d'une grande richesse historique constituent les véritables enjeux des mesures; on voit que le programme Stadtumbau Ost combine deux objectifs : stabiliser le marché du logement et améliorer l'attractivité des villes.

De telles mesures ne peuvent être valables à long terme que si elles sont conçues de manière globale: c'est pourquoi l'Etat a souhaité encourager la mise en place de concepts urbanistiques de développement (städtebauliche Entwicklungskonzepte) ${ }^{3}$. Ces concepts doivent garantir que le programme de rénovation de chaque ville s'appuie sur une analyse des besoins en logements dans le futur, que les mesures prévues correspondent aux objectifs du programme et que ne seront reconstruits que des bâtiments nécessaires d'un point de vue urbanistique ou économique. Afin d'encourager les communes dans la constitution de ces concepts, le gouvernement fédéral a choisi de consacrer une partie des aides à la mise en place d'un concours pour récompenser les meilleurs d'entre eux. Ce concours s'est déroulé de novembre 2001 à septembre 2002. La même année, quelques progrès ont déjà été enregistrés grâce aux démolitions entreprises dans le cadre du programme Stadtumbau Ost, puisque la moyenne du nombre de logements vides dans les sociétés immobilières municipales et les coopératives immobilières est passée de $16 \%$ en 2003 à 14,9\% en 2004.

21 Les voies indiquées par le programme Stadtumbau Ost pour rendre aux villes une certaine attractivité ont sans doute de quoi surprendre puisqu'il insiste sur la nécessité de « rétrécir » les villes. Comme on l'a vu, les conditions sont inédites; les réponses à apporter le sont donc forcément tout autant. Les communes prioritaires pour l'attribution des aides dans le cadre du programme Stadtumbau Ost sont celles qui présentent un taux de logements vides supérieur à la moyenne du Land. C'est ainsi qu'entre 2002 et 2004297 communes ont bénéficié du programme.

Un bilan des premières années du programme, réalisé en 2006 par le ministère fédéral des Transports, de la Construction et de l'Urbanisme (BMVBS, 2006), insiste pour le cas des quartiers de Plattenbau sur le fait que de nombreuses communes ont sous-évalué les 
démolitions nécessaires. Il est rappelé qu'il n'est pas souhaitable de s'appuyer de manière trop accentuée sur les désirs des habitants actuels dans la mesure où ceux-ci sont souvent âgés, et que ce type de quartier apparaît aujourd'hui répulsif pour les jeunes générations. En dépit de l'importance des quartiers centraux pour la revitalisation des villes, les communes ont investi beaucoup de moyens dans les grands ensembles. Dans le cadre du programme, elles ont dû délimiter des zones d'actions, qui recevraient les aides : $40 \%$ ont été situées dans des quartiers de Plattenbau et $25 \%$ dans le tissu urbain datant d'avant 1948. Les villes ont pu considérer en effet que les démolitions dans les quartiers périphériques entraineraient mécaniquement un report des habitants et des investissements sur les quartiers anciens, ce qui ne s'est produit que dans peu de cas. Joue aussi le fait que dans les grands ensembles, les propriétaires sont moins nombreux et mieux connus (les sociétés immobilières en particulier), ce qui facilite la mise en place des actions.

Quelques aspects de la politique urbaine en Allemagne peuvent être mis en avant afin de mieux saisir le cadre dans lequel s'inscrit le programme Stadtumbau Ost: contrairement à la tradition centralisatrice de la France, la politique urbaine relève habituellement des compétences des villes elles-mêmes, ainsi que des Länder ${ }^{4}$. Ce programme, on l'a dit, combine urbanisme et politique du logement ${ }^{5}$, ce qui va dans le sens des évolutions souhaitées par le gouvernement fédéral : les politiques urbaines jusqu'alors portaient uniquement sur le tissu urbain lui-même, et l'on cherche à l'heure actuelle à mettre en œuvre des politiques intégrées. Par ailleurs, on veut que la rénovation ne soit pas considérée comme un ensemble de subventions, mais comme une nécessité de développement, un devoir indispensable pour les villes.

\section{B. Une réponse adaptée ? Stadtumbau als Chance}

Nous reprenons ici le titre de l'article de Rüdiger Pohl (2003), lequel, analysant la situation actuelle de la Saxe-Anhalt et élargissant sa problématique à l'ensemble des nouveaux Länder, considère le Stadtumbau comme la seule issue possible à la situation urbaine actuelle des nouveaux Länder: espérer une augmentation de la population dans les années à venir est largement une «illusion». Il faut donc affronter la situation actuelle pour ne pas entrer dans un cercle vicieux. La transformation envisagée des villes doit être considérée comme un « investissement». R. Pohl juge cependant que les moyens engagés restent insuffisants. Il soulève en outre plusieurs questions qui rappellent, si besoin est, la gravité de la situation et les défis à affronter : que faire si la population continue de décroître? Y aura-t-il alors une nouvelle vague de démolitions? Comment la financer? La Saxe-Anhalt pourra-t-elle inscrire ses efforts dans la durée et assurer à toutes ses villes une aide financière? Cela n'est guère envisageable. De plus, la densité de population est relativement modérée dans ce Land et ce phénomène ne peut que s'accroître: le maintien des infrastructures sera donc toujours plus coûteux. Quelles communes feront les frais de ce rétrécissement des moyens, et quelles entreprises de logements? Ces questions doivent être abordées aujourd'hui par ceux qui dirigent le programme, car il n'est pas réaliste de penser que les moyens seront suffisants pour apporter une aide à chaque commune et à chaque entreprise de logement. Tôt ou tard, la concentration des moyens sera inévitable.

R. Pohl souligne également à quel point la manière d'aborder le thème de la transformation des villes doit être encore corrigée. Perçu en tant que thème 
typiquement est-allemand, il devrait au contraire être considéré comme concernant l'ensemble du pays. La population en effet est en passe de décroître également dans les anciens Länder, quoique la situation soit loin d'être comparable. Certes, on se préoccupe déjà de ce problème, mais il serait judicieux de considérer la transformation des villes comme « un défi global allemand ». Cette transformation des villes doit être l'occasion d'un changement dans la façon d'aborder l'urbain. Si à l'heure actuelle on mesure une politique urbaine à l'aune de sa capacité à attirer les activités et les habitants, notamment par l'offre sociale et culturelle, avec une population en diminution, il faut investir dans une optique de limitation des conséquences négatives : ce sont des "investissements sans capitalisation positive». Il faudrait que cette mutation des conceptions soit bien intégrée dans les nouveaux Länder, déjà concernés par une situation de déclin, afin de pouvoir ensuite gagner les régions occidentales.

Le programme de rénovation urbaine Stadtumbau Ost qui doit permettre une restructuration et une adaptation du tissu urbain au contexte de fort déclin démographique qui marque les nouveaux Länder s'applique de manière différenciée en fonction de la taille et des caractéristiques des villes. Pour illustrer cela, on montrera comment il doit être mis en place dans deux villes nouvelles aux caractéristiques bien différentes: d'une part, la ville d'Eisenhüttenstadt implantée en 1951 autour d'un complexe sidérurgique créé sur l'Oder, à la frontière polonaise ; d'autre part, Neustadt fondée en 1963 aux portes de la grande ville de Halle pour loger les ouvriers de l'industrie chimique située au sud de la ville. Les deux villes se différencient d'abord par leur taille, puisque Eisenhüttenstadt a atteint au maximum 50000 habitants, alors que Neustadt avait été prévue pour loger environ 100000 habitants. Elles se différencient plus encore par leur situation, Eisenhüttenstadt étant une ville monoindustrielle en situation isolée et périphérique, alors que Neustadt apparaît comme la banlieue d'une grande ville, Halle, à laquelle elle a d'ailleurs été administrativement rattachée en juin 1990.

\section{Eisenhüttenstadt, la ville idéale du socialisme face aux réalités de l'après réunification}

Ce premier exemple offre la possibilité de saisir avec netteté les différentes étapes de l'évolution de l'urbanisme en RDA, et par là, de mieux comprendre certaines problématiques urbaines actuelles. Ce cas particulier est également l'occasion d'étudier l'application du programme Stadtumbau Ost à l'échelle d'une ville moyenne. On notera que différents programmes s'entrecroisent pour aider les communes à regagner en vitalité.

Ville modèle, son destin s'est trouvé fortement lié à celui du pays. Eisenhüttenstadt avait vocation à loger les habitants du récent combinat sidérurgique qui devait fournir l'acier nécessaire à la reconstruction du pays. La ville devait donc être la vitrine du nouvel ordre économique, social et politique. Les premiers plans prévoyaient quatre complexes, soit quatre quartiers d'habitation comportant chacun les équipements et services nécessaires à la vie quotidienne. Les directives architecturales et urbanistiques étaient dictées par le pouvoir stalinien, et ont engendré un ensemble exemplaire de la « construction dans la tradition nationale ». Déjà le troisième complexe ne répond plus que partiellement à ces directives; quant au quatrième, il correspond déjà à une nouvelle étape, celle de la construction en série. Le développement du combinat 
sidérurgique entraînant des besoins toujours plus grands en main-d'oeuvre, de nouveaux complexes s'ajoutèrent progressivement au premier ensemble urbain. Du quatrième au septième complexe se lisent tant les évolutions de l'industrialisation de la construction que les difficultés croissantes du pays: le complexe IV présente des bâtiments de taille encore réduite, alors que les complexes V et VI montrent une augmentation du nombre d'étages et des bâtiments de plus en plus longs. Le complexe VII est caractérisé par une dégradation de la production du bâti, mais aussi des aménagements : les équipements et services sont loin d'être en suffisance.

Ce rapide panorama de l'histoire de la ville montre à quel point y sont lisibles les étapes de l'histoire urbaine de la RDA, et les différences urbanistiques et architecturales établies suivant les générations de bâtiments se retrouvent aujourd'hui dans le destin différent des quartiers et le traitement qui leur est réservé dans le cadre des transformations actuelles.

Figure 2 : le plan de rénovation de Eisenhütenstadt

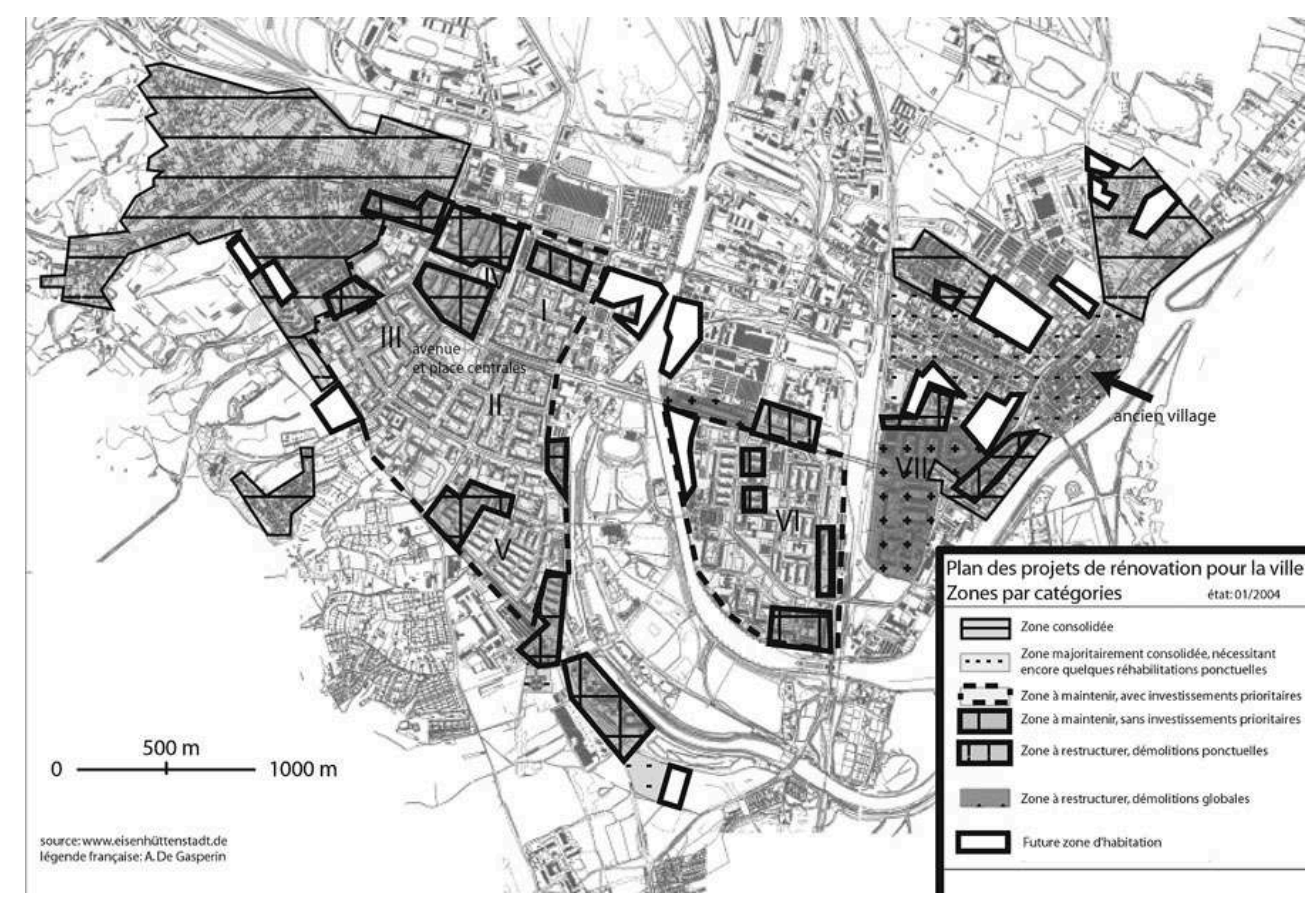


Photo 1 : La démolition de la partie sud du complexe VII à Eisenhüttenstadt

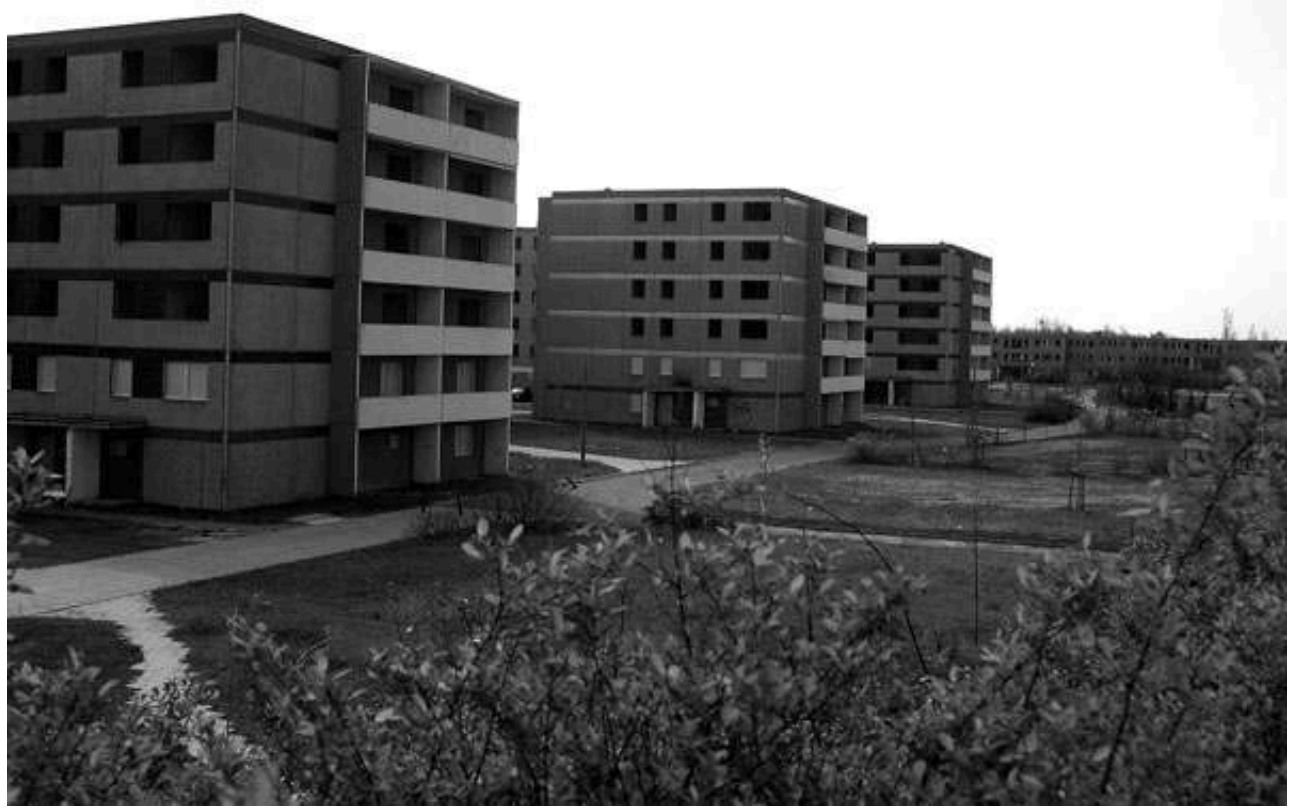

A. De Gasperin, 2005 réunification : elle a perdu $32 \%$ de sa population entre 1989 et 2004 , où elle ne comptait plus que 35773 habitants. Depuis 2001, 500 logements par an se vident. Les deux principales sociétés immobilières de la ville (elles possèdent $85 \%$ des logements) avaient en moyenne en novembre $200425 \%$ de logements inoccupés, en dépit des démolitions déjà effectuées. Le complexe VII a été relativement le plus touché par les pertes de population : depuis 1995, il a perdu $57 \%$ de sa population. Il présente les plus forts taux de logements inoccupés : $49 \%$ en avril 2004 , soit $84 \%$ pour le quartier Sud et $35 \%$ pour le quartier Nord. Dans le cadre du programme Stadtumbau Ost, la partie sud du septième complexe a donc été totalement démolie en 2006, soit 1150 logements (fig. 2 et photo 1). Entre 2006 et 2015, ce sont 1650 logements sur 2000 que compte la partie nord du quartier qui vont disparaître. Ces démolitions ponctuelles ou à l'échelle d'un quartier (comme le montre le cas du complexe VII) ouvrent la question des friches urbaines : l'absence d'entretien de certaines zones, l'impossibilité de leur redonner une fonction entraîne souvent une dégradation rapide qui nuit à l'esthétique de la ville. Dans le complexe VII, une renaturation (Renaturierung) est prévue. Notons que ce terme, comme le rapporte le bilan effectué en 2006 masque souvent le fait que ces espaces ne retrouveront tout simplement pas d'usage.

31 Cependant, alors que l'on démolit une partie des complexes d'habitation les plus récents, les trois premiers complexes et l'avenue des Peupliers (l'avenue principale) sont considérés comme des zones à réhabiliter. Leur situation est quelque peu particulière, puisqu'ils sont classés monument historique (Denkmalbereich) depuis 1984. La décision de classer puis de réhabiliter après la réunification cet ensemble urbain traduit la valeur historique de cet objet. On ne peut parler à leur endroit de "grands 
ensembles ", dans la mesure où la construction des immeubles n'ayant pas été faite de manière industrialisée, ils ne présentent pas la monotonie caractéristique des grands ensembles; de plus, les principes de la ville moderne étaient encore bannis, et ces quartiers offrent une trame urbaine assez traditionnelle, notamment avec la conservation de la rue, et des bâtiments qui ne dépassent pas quatre étages. La réfection de nombreuses façades a permis de remettre en valeur la qualité architecturale de ce noyau urbain. Tous les logements ne feront toutefois pas l'objet d'une réhabilitation. L'image de la ville et, partant, son attractivité sont fortement dépendantes de la qualité paysagère de ces complexes. En tant que centre-ville, le soin apporté à l'entretien des bâtiments est décisif, mais c'est en même temps l'entretien du patrimoine historique de la ville qui se joue dans ces réhabilitations. Dans le complexe $\mathrm{V}$ qui lui, au contraire, répond à la définition d'un grand ensemble, les travaux sur l'aspect extérieur des immeubles ont permis de diminuer l'impression de monotonie et d'anonymat. Ce travail sur l'aspect extérieur des immeubles a une importance capitale quant à la qualité de vie que peut offrir la ville. Cela ne peut que favoriser l'appropriation de l'espace par les habitants. Or, la problématique est telle dans toute opération de réhabilitation d'immeubles : redonner à l'espace la possibilité d'être un lieu d'habitation au sens plein du terme, c'est-à-dire où la population peut avoir envie d'habiter. Le complexe VI a également fait l'objet de nombreuses mesures, dans le cadre d'un programme, Zukunft im Stadtteil (Un Avenir pour le Quartier) lancé par le Land du Brandebourg. L'ensemble de la ville est donc concerné par des mesures de réhabilitation; il s'agit tant de l'extérieur que de l'intérieur des logements (photo 2). Les travaux menés jusqu'ici et ceux planifiés pour les années à venir ont une ampleur difficile à imaginer dans un autre pays. Il faut transformer des quartiers entiers de bâtiments identiques, souvent vieillis, aux espaces verts dégradés, à la qualité réduite par la hâte de la construction, en véritables espaces d'habitation. 
Photo 2 : Exemple de façade d'immeuble réhabilitée dans le complexe VI de Eisenhüttenstadt

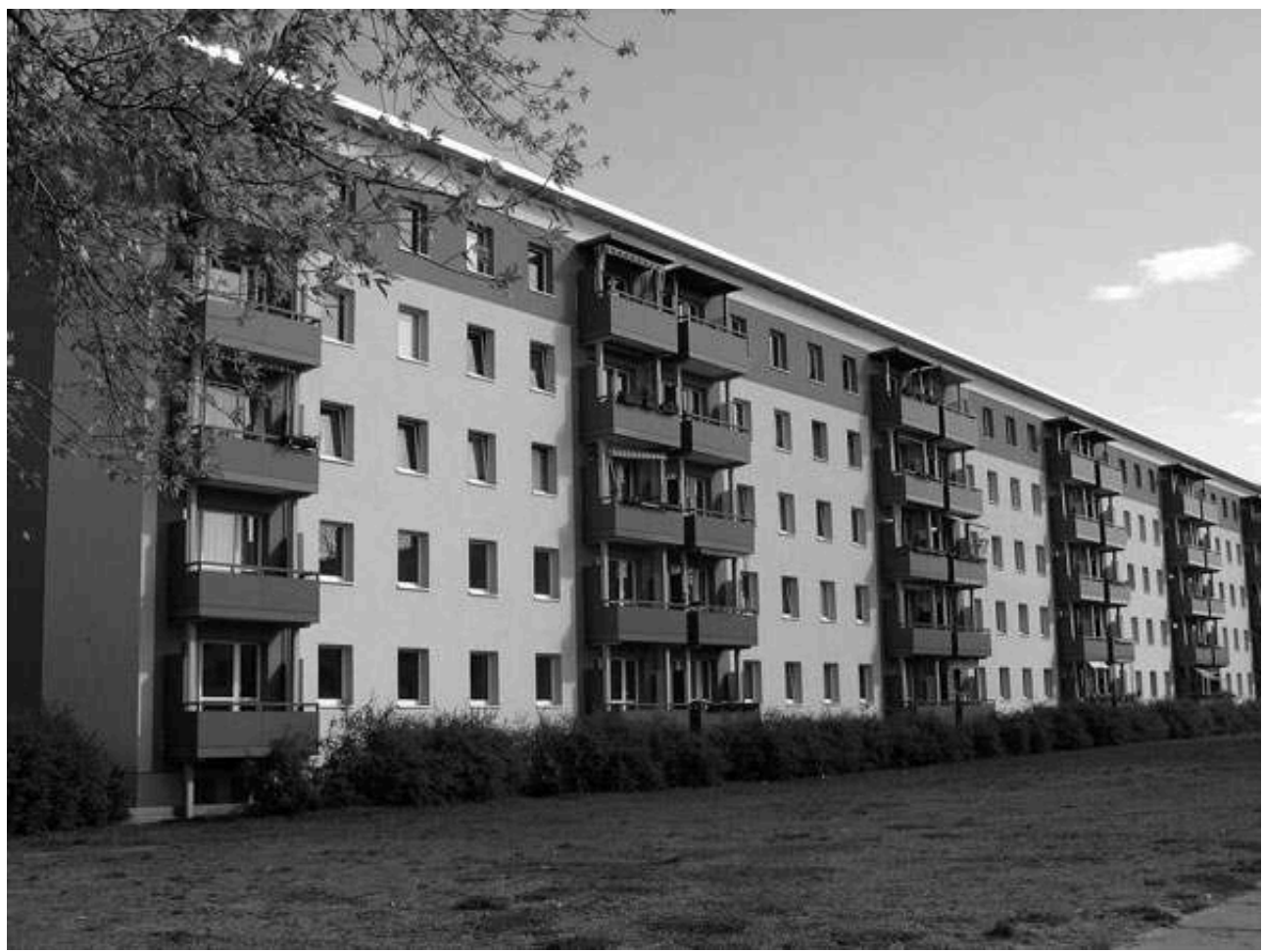

A. De Gasperin, 2005

32 Eisenhüttenstadt a participé au concours Stadt 2030 lancé en 2000 par le Ministère fédéral pour l'Education et la Recherche consistant à proposer un concept global de développement pour préparer l'avenir et définir une image de la ville. Parmi les 109 communes et régions urbaines de l'ensemble de l'Allemagne qui ont participé à ce concours, Eisenhüttenstadt a fait partie des 21 villes qui se sont vues récompensées pour leur projet. L'image est un moyen pour la ville de se démarquer et d'affirmer une identité propre. Elle lui permet de se positionner sur le marché de la concurrence, en vue d'attirer investisseurs et nouveaux habitants. C'est aussi un moyen offert aux anciens habitants pour raffermir leur lien avec leur ville. Eisenhüttenstadt met en avant la richesse historique de toute la région, avec la particularité d'un cœur urbain exceptionnel en Allemagne. Le potentiel touristique historique est complété par celui de la voie d'eau. La commune mise également sur son noyau industriel, elle valorise sa tradition et son savoir-faire industriels, afin d'attirer les entreprises. La ville cherche à attirer particulièrement les jeunes familles (par exemple en adaptant les horaires des crèches aux horaires de travail), tout en insistant sur sa capacité à accueillir tous les groupes d'âge. Prenant acte du vieillissement de la population, elle souhaite adapter ses logements et les espaces de rencontre. Elle se présente enfin comme un centre à l'échelle locale et veut offrir un maximum de services, tant commerciaux que culturels ou sociaux. Un article du Monde Diplomatique analyse le modèle proposé en ces termes: "La vision que les urbanistes d'Eisenhüttenstadt développaient était moins [prometteuse]: difficile de vendre l'idée d'une ville de retraités aux habitants d'une cité qui était la plus jeune de la RDA. Tout cela a fini en patchwork industriel, touristique et culturel au service d'une politique favorable à la vie familiale et la revalorisation de l'histoire » (Linden, 2004). 


\section{Halle-Neustadt : restructuration d'une ville nouvelle socialiste}

Halle-Neustadt (fig. 3) fait partie des quatre plus grands quartiers de Plattenbau de l'ancienne RDA, avec Marzahn et Hellersdorf à Berlin, et Grünau à Leipzig. Ces grands ensembles atteignent la taille de grandes villes. On peut cependant souligner que, contrairement à Neustadt, les trois autres quartiers n'avaient pas vocation à être des villes autonomes, mais bien des quartiers intégrés à des centres urbains préexistants. Nous pouvons à partir de l'exemple de Halle-Neustadt présenter un autre aspect des transformations actuellement en cours dans les villes des nouveaux Länder: la complexité de l'application du programme Stadtumbau Ost. La nécessité de se placer à plusieurs échelles à la fois, la compétition entre les quartiers, les modèles urbains sont quelques uns des éléments qui alimentent et éclairent tout à la fois cette complexité.

Figure 3 : les étapes du développement de Halle

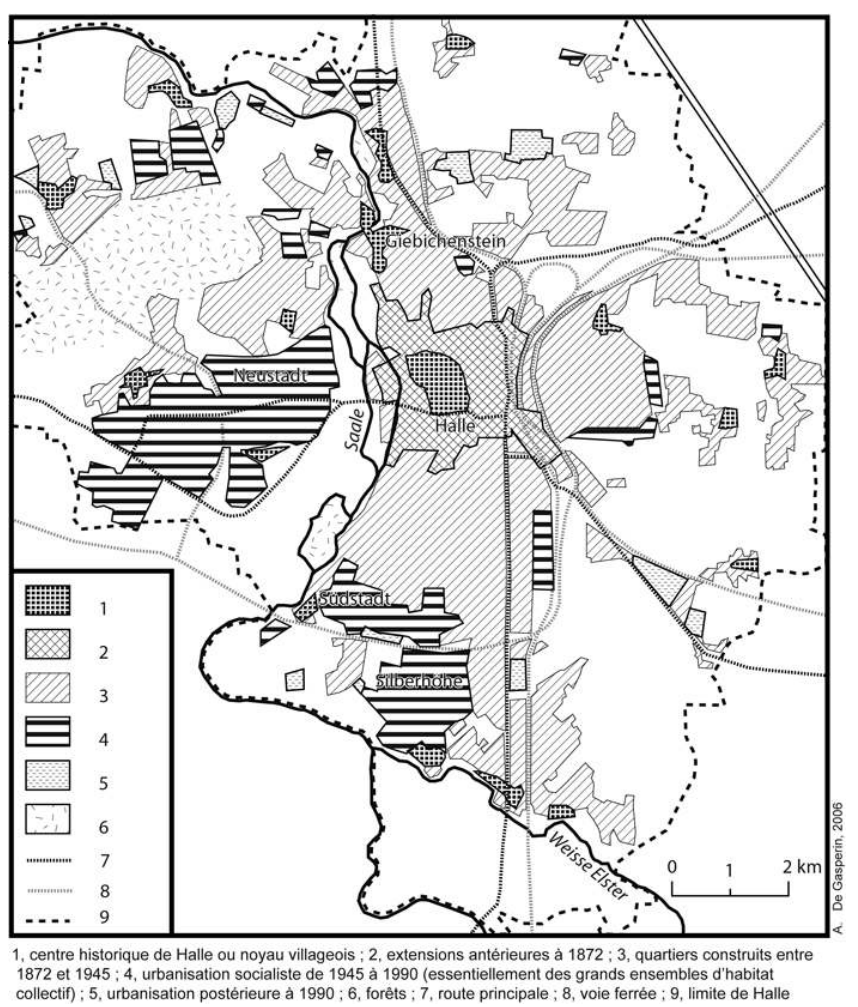

Lorsque a été lancé le programme Stadtumbau Ost, le ministère fédéral des Transports, de la Construction et du Logement a proposé une synthèse (BMVBW, BBR, 2001) pour aider les communes à établir leur concept intégré de développement urbain (integriertes Stadtentwicklungskonzept). Cette présentation consacre une réflexion aux différentes possibilités existantes pour réaliser les démolitions au sein des quartiers. Première option: une dédensification, permettant l'utilisation des surfaces libérées en stationnement ou espaces verts. Une amélioration du cadre de vie est ainsi rendue possible. Cette dédensification peut se faire soit par la démolition de quelques immeubles, soit par la démolition partielle de barres, ou encore la diminution du nombre d'étages pour certains bâtiments. Seconde option : une démolition réalisée sur une vaste surface ; on envisage de recourir à une telle extrémité dans des quartiers où 
le fonctionnement du marché immobilier est gravement compromis. Là encore, plusieurs modalités sont proposées : réduction en périphérie, disparition du cœur du quartier ou démolition totale d'un complexe au sein du quartier. Troisième option : le maintien d'un bâti inoccupé. Cette alternative est envisagée surtout si manquent les moyens pour la démolition.

La mise en place d'une stratégie au sein des quartiers s'inscrit au sein d'une réflexion assez semblable à l'échelle de la ville. Dans le cas de Halle/Saale, le concept de développement urbain a évalué trois modèles possibles de développement: la "ville des inclusions vertes (perforation ponctuelle) », la « ville des îles » (repli concentré), la « ville des coulées vertes » (mise en connexion de la ville et de l'espace rural) ».

À l'échelle du quartier Neustadt les interrogations sont celles-ci : faut-il conserver tous les complexes, démolir quelques immeubles dans chacun et ainsi les dédensifier, ou, au contraire, démolir les complexes périphériques et conserver le cœur du quartier, ou encore préserver la structure générale et le fonctionnement de Neustadt? Chaque éventualité comporte avantages et inconvénients: dans bien des cas, la solution la moins coûteuse est de démolir les barres ou les tours vidées de leur population. Mais cette solution en apparence simple engendre une multitude de problèmes techniques : il est par exemple délicat de démolir entièrement un complexe d'habitation si une entreprise y a la majorité de ses logements, car leur disparition impliquerait la mise au chômage des employés, sans parler bien sûr des problèmes sociaux ou même psychologiques des habitants qui voient leur quartier progressivement disparaitre. Il est à noter que les habitants ne sont pas forcément hostiles à l'idée de quitter leur logement, beaucoup plus cependant à celle de quitter le complexe : ils ont en effet souvent noué des relations de voisinage, et ont leurs habitudes dans ce complexe. Pour toutes ces raisons, la démolition complète d'un complexe est difficile à envisager. Les entreprises immobilières qui décident de faire disparaître un immeuble doivent tenir compte chacune des projets des autres entreprises immobilières et de la commune : par exemple, la commune peut choisir de transformer une école qui n'est plus utilisée, ce qui influe sur la qualité de vie du quartier. On peut à titre indicatif évoquer le cas d'un autre quartier de Halle/Saale, Silberhöhe, pour lequel a été retenue l'option de la villeforêt (Waldstadt) : la démolition d'immeubles au cœur du quartier a entraîné une redéfinition de cet espace central.

Dans les réflexions conduites sur les stratégies possibles de réaménagement des quartiers, le bilan mené à l'échelle de l'ensemble des villes en 2006 souligne que le choix de démolitions ponctuelles en vue d'une dédensification (on parle souvent de perforation, que ce soit à l'échelle d'une ville ou d'un quartier) augmente le risque de déliquescence de la structure urbaine. Le terme «perforation » peut être employé dans un sens connoté soit positivement, soit négativement; dans le premier cas, il désigne un modèle possible d'évolution de la structure d'une ville ou d'un quartier, avec des dédensifications ponctuelles du tissu urbain. Il est alors une option choisie par la commune. Sinon, il est synonyme de désagrégation des structures urbaines. Cependant, comme on vient de le dire, les communes ont été mises en garde contre ce modèle de développement, qui, même choisi, peut néanmoins être source de difficultés, d'autant plus que cette option entraîne pour la ville un accroissement des coûts de fonctionnement. 50293 en 2005, soit seulement 55 \% de la population de 1989. Aujourd'hui, 21,6 \% de la 
population de Halle vit à Neustadt (Fachbereich Stadtentwicklung und-planung, Ressort Stadtplanung, 2006). On peut établir une comparaison avec le quartier de Silberhöhe (fig. 3), dont la situation est la plus préoccupante à Halle. Il comptait 18000 habitants en 2004, soit $46 \%$ de la population de 1990. Deux tiers des démolitions déjà réalisées à Halle en 2004 l'ont été dans ce quartier. Des conceptions de la ville moderne, HalleNeustadt a hérité une structure hiérarchisée : chaque complexe est organisé autour d'un petit pôle, et le quartier dans sa totalité s'articule à partir d'un centre, la séparation des fonctions, un réseau de circulation piétonne indépendant des voies automobiles. Environ $50 \%$ de la surface du quartier est consacrée à des espaces verts. La configuration des zones vertes n'était pas achevée au moment de la réunification, ainsi que la construction du centre-ville, et il manquait les équipements commerciaux de certains complexes. Malgré ces lacunes, les urbanistes estiment que Neustadt, contrairement à d'autres grands ensembles, est un quartier de qualité, avec une structuration solide et un bon niveau d'équipement, ce qu'il doit à son statut de villemodèle. De grandes surfaces libres, souvent vertes, un bon niveau d'équipements sportifs et ludiques (un cinéma, une piscine et un ensemble sportif complet), de larges voies de circulation et de nombreuses possibilités de garage sont cependant contrebalancés par une offre de logement peu diversifiée, la monotonie et une orientation difficile, le manque de différenciation entre public et privé dans les espaces non bâtis et la faiblesse de leur composition paysagère, ainsi que par la démesure de certaines voies de circulation.

La décennie 1990 a été marquée par des réalisations importantes, qui ont permis de compléter certains des projets qui n'avaient pu être menés à bien du temps de la RDA. La structure demeurée inachevée du centre-ville a été complétée par le "Carré de l'avenue Magistrale» (Magistralen-Carree), qui regroupe un hôtel, des services administratifs, des commerces; en 2000 a été ouvert un centre commercial. L'année précédente, on a inauguré la ligne de tramway reliant le quartier au centre ancien de Halle: elle améliore de manière significative la liaison du quartier au reste de l'ensemble urbain. Une proportion importante d'immeubles a fait l'objet de réhabilitations totales ou partielles (photo 3): dans l'espace central de Neustadt, une très forte part des logements a été totalement modernisée. Au contraire, les complexes V, VI et la zone d'habitation Am Südpark présentent un fort taux de logements non, ou seulement partiellement, réhabilités (fig. 4). 
Figure 4 : plan de rénovation de Halle-Neustadt

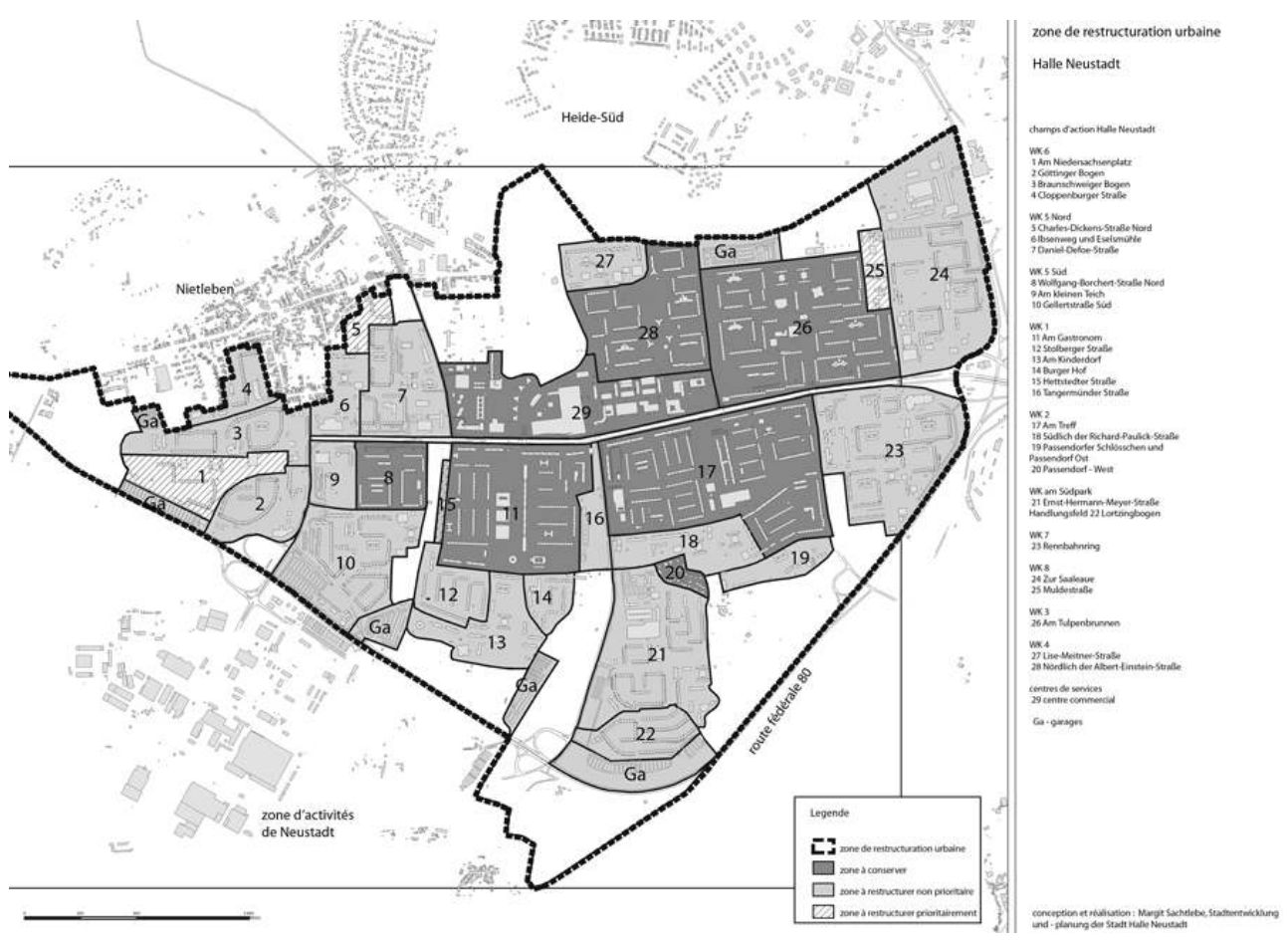

Photo 3 : Vue du complexe 8, en bordure est de Neustadt

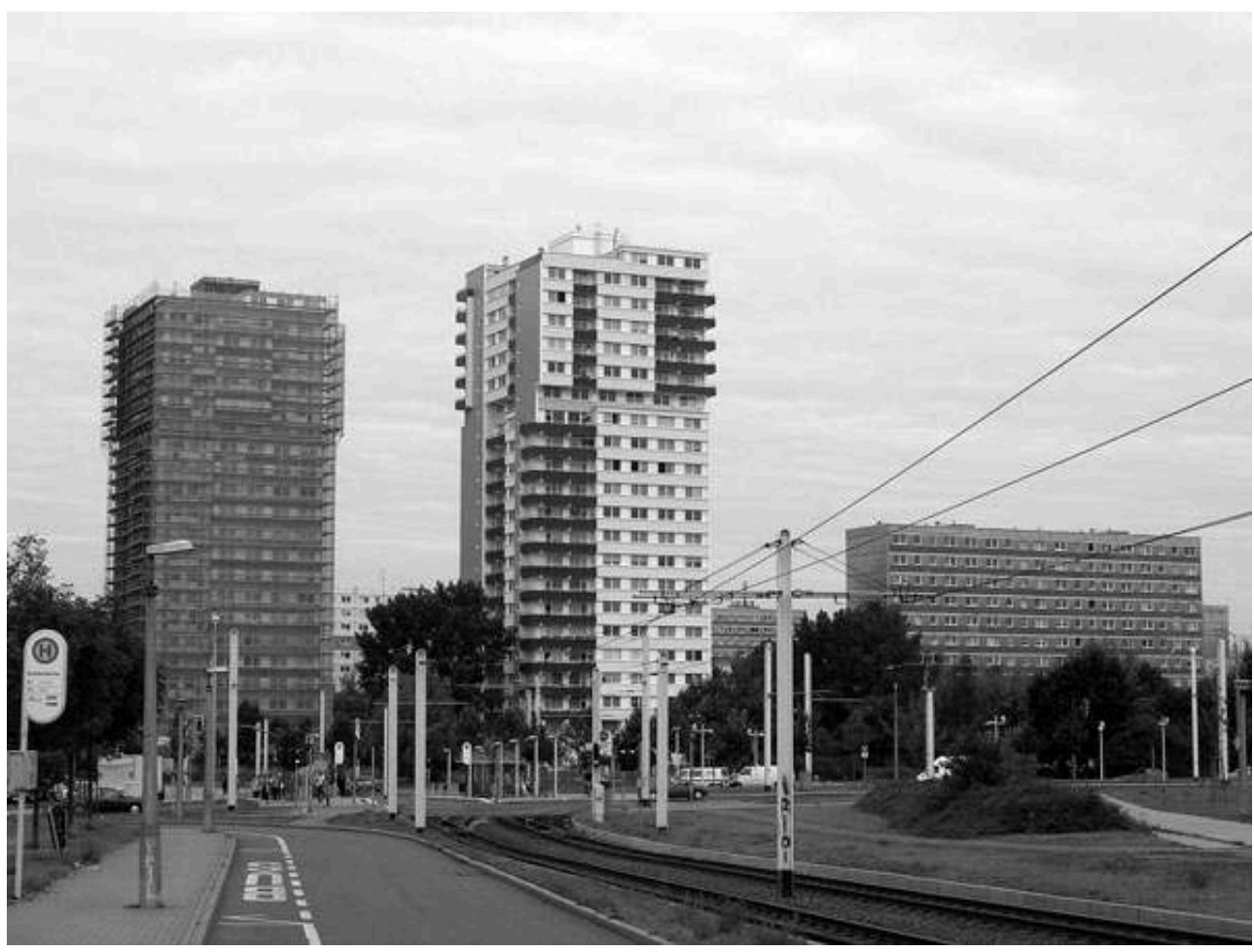

La vue présente deux tours de 20 étages, dont l'une a été réhabilitée, alors que pour l'autre les travaux sont en cours. Au premier plan, la ligne de tramway qui relie Neustadt au centre de Halle. La proximité du centre assure la stabilité de la population habitant dans le complexe 8.

M. Deshaies, 2005 
Cette présentation des réalisations menées dans les années 1990 ouvre celle des opérations qui ont été ou vont être menées entre 2000 et 2010, dans le cadre du processus de rénovation urbaine. D'aucuns estiment, au regard des sommes investies dans la modernisation du quartier, qu'une partie de ces investissements a été réalisée en pure perte, puisque cela n'a pas permis d'enrayer les départs de population, et qu'aujourd'hui l'on semble s'orienter dans une logique inverse de démolitions. Certes, démolitions et modernisations semblent contradictoires, et il faut reconnaître que certains bâtiments réhabilités présentent aujourd'hui des taux élevés d'inoccupation. Cependant, la population de Neustadt est toujours celle d'une ville moyenne, et certains équipements restés inachevés en 1990 étaient indispensables. Se joue en fait ici un débat entre ceux qui estiment que Neustadt ne devrait pas faire l'objet d'investissements aussi importants, au vu de son évolution démographique en comparaison du reste de la ville: car dans la mesure où les moyens financiers demeurent très limités, il y a forcément concurrence entre les quartiers; devraient être privilégiés ceux qui sont attractifs, ou le centre dont la richesse historique et le rôle fondamental dans l'image et l'attractivité de Halle sont indéniables. Dans une ville menacée par la perforation, mieux vaudrait se recentrer sur les quartiers centraux. Le point de vue contraire insiste sur le fait qu'à Neustadt vit encore la population d'une ville moyenne, qui a droit à un quartier offrant une bonne qualité résidentielle; de plus, les investissements réalisés peuvent atténuer une trop forte diminution de la population. L'idée est également de rendre le quartier suffisamment attractif pour que les populations s'y installent par choix et non par contrainte financière, et limiter ainsi une ségrégation socio-spatiale au sein de Halle.

41 Le débat qui se joue à Halle est représentatif de la situation de nombreuses villes des nouveaux Länder. Le bilan à mi-parcours réalisé par le ministère fédéral des Transports, de la Construction et de l'Urbanisme (BMVBS, 2006) insiste sur le fait que dans un contexte de déclin, des investissements mal réalisés auraient des répercussions plus lourdes encore que dans une situation urbaine classique. L'accent est mis également sur la nécessité de mettre en valeur les quartiers anciens, qui donnent à la ville son image ; on retrouve là d'ailleurs une tendance générale en Europe occidentale de revitalisation des centres anciens. Les grands ensembles ne paraissent pas à même d'assurer l'avenir des villes, c'est pourquoi le ministère fédéral des Transports, de la Construction et de l'Urbanisme met en garde les communes et les entreprises immobilières contre une mauvaise appréciation de ces faiblesses réelles.

Dès 1997, des actions préparatoires ont été lancées, en vue de mettre en place des structures de travail en commun, ce qui aboutit à la création du Réseau Rénovation urbaine (Netzwerk Stadtumbau) et du Groupe d'orientation Rénovation urbaine (Lenkungsgruppe Stadtumbau). Le premier a été mis en place par les entreprises et coopératives immobilières, et fait participer également d'autres acteurs dont le devenir économique est lié au développement des quatre grands ensembles de Halle. Le second, fondé par la ville, a pour objectif de réunir tous les acteurs concernés autour de la question de la rénovation, afin de mettre en place un concept de développement qui soit travaillé et accepté par tous.

43 Ces organisations ont permis de définir un concept de développement pour la ville : il comprenait une analyse de la situation démographique et du marché du logement de 1990 à 2000 et des prévisions pour 2010, ainsi que des scénarii de développement de l'espace urbain, de développement par quartier, avec leur classement en trois 
catégories : zone à restructurer, zone à maintenir, zone stable. A cela s'ajoutait une présentation du processus de rénovation: instruments, gestion, rôle des entreprises immobilières. Cette "conception de développement urbain" était accompagnée de deux « concepts de réaménagement » (Neuordnungskonzepte) pour les quartiers Neustadt et Silberhöhe.

Le concept de réaménagement de Neustadt privilégie l'idée d'une dédensification qui se fasse de manière concentrée dans certaines parties du quartier, plutôt qu'un éclatement des mesures de démolition dans l'ensemble des complexes. Les complexes I et II souffrent moins des pertes de population. Plusieurs explications peuvent être avancées : plus anciens, ils offrent un bon niveau d'équipement, et des densités qui restent raisonnables; la population y est aussi plus âgée, d'où une mobilité plus faible. Les zones centrales apparaissent donc plutôt comme stables, et l'on a choisi l'option de leur renforcement, avec une légère dédensification. On cherche plutôt à mener les démolitions dans les zones périphériques, notamment vers le sud, où passe une voie importante de circulation (B 80): ainsi la distance serait plus grande entre les habitations et les nuisances de la route. De même, on envisage d'agrandir la zone verte qui s'étend en direction de la Saale, et est située dans le lit majeur du cours d'eau, donc en zone inondable. On espère, grâce à l'extension des espaces verts en périphérie, atténuer la brutalité de la rupture entre le quartier et son environnement, très caractéristique des grands ensembles. La ligne directrice ici développée montre que l'on souhaite conserver la structure fondamentale du quartier, avec une articulation des complexes autour d'un centre; de même, au sein des complexes, l'objectif est de maintenir une organisation autour du noyau. Cependant, la forte diminution de la population ne permet pas le maintien de tous les centres : c'est pourquoi il faut revoir la manière dont est définie l'organisation des pôles.

La première phase prévue par le concept de réaménagement devait conduire à la démolition de 2000 logements entre 2001 et 2005, et au total à la disparition de 4500 logements. Sont avant tout visés les immeubles de 17 étages (photo 4). Cette ligne directrice a en outre vocation à guider les investissements. On peut noter que l'une des difficultés que rencontrent les urbanistes dans leur travail est de devoir se fonder sur des pronostics de population, et ce à tous les niveaux ; dans le cas de Halle-Neustadt, il faut chercher à évaluer quelles sont les tendances, complexe par complexe, pour mettre en place une stratégie.

Cette ligne directrice montre que l'on cherche à corriger certaines déficiences inhérentes à la forme urbaine en tant que telle, mais tout en conservant le fonctionnement général du quartier. Ce choix du maintien de la structure de base s'explique à la fois par l'accoutumance des habitants, surtout les plus anciens à certains aspects de la ville moderne, comme la séparation des circulations, et par le fait que l'identité de Halle-Neustadt passe par son caractère de ville moderne. 


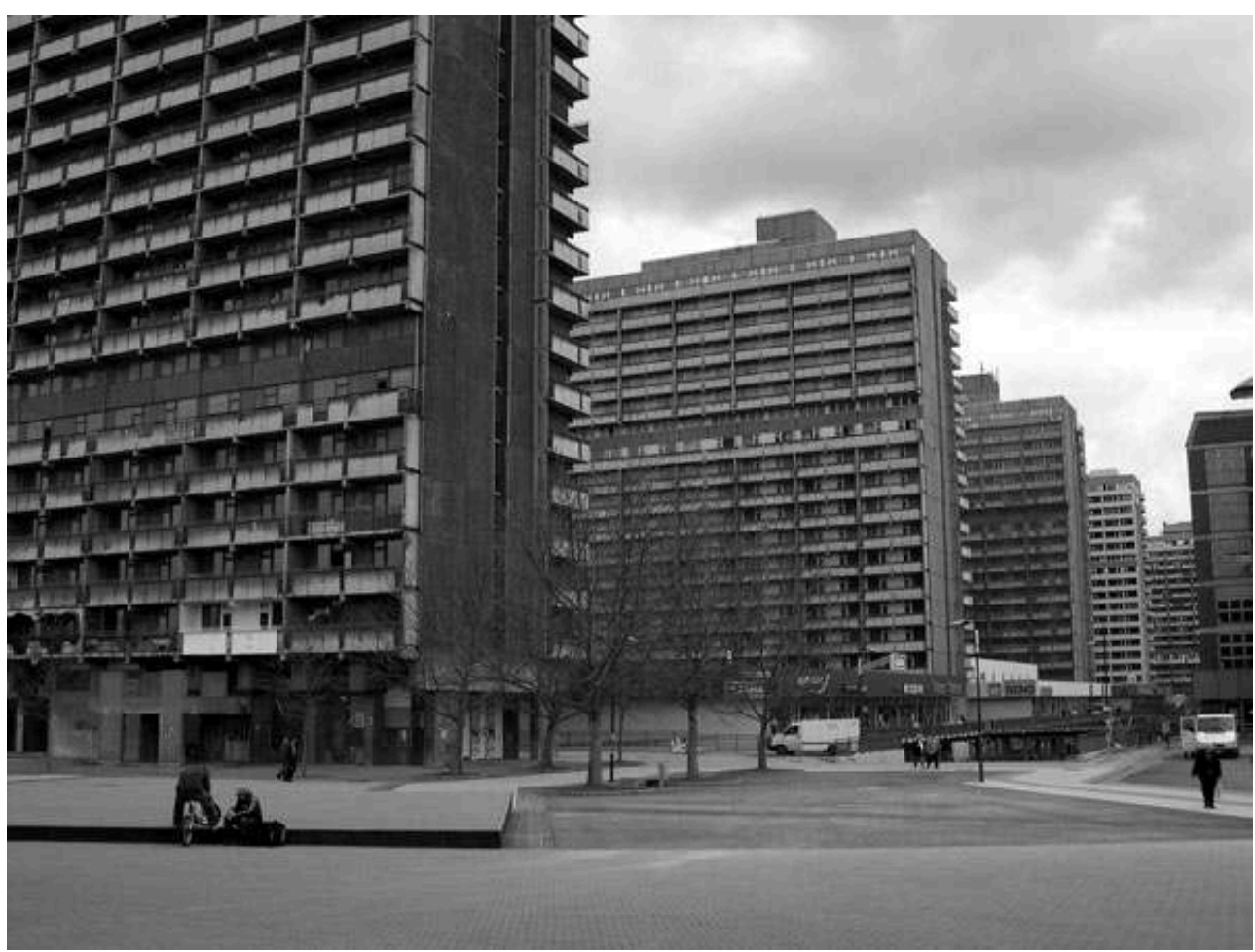

Le quartier très dense, présente plusieurs immeubles de 17 étages actuellement vides, qui doivent être démolis.

A. De Gasperin, 2006

Les enjeux à l'heure actuelle pour Halle-Neustadt sont une diminution des densités bâties, une diversification de l'offre de logements, la réduction du nombre de logements vides et la reconfiguration des espaces verts. D'ici à 2010 est projetée la démolition d'environ 5000 logements (y compris les démolitions déjà effectuées). Quatre complexes présentent de lourds handicaps: le complexe VI, les complexes V Nord et V Sud et le complexe Am Südpark. Ils sont particulièrement concernés par les démolitions. Les handicaps du complexe VI tiennent à sa situation périphérique, dans Halle-Neustadt, mais aussi par rapport à l'ensemble de Halle, et à une modernisation restée très partielle des immeubles. Le complexe Am Südpark est le dernier né de HalleNeustadt, dans les années 1980. Les immeubles, originellement prévus à 5 étages, en ont finalement reçus 6 , en raison d'un besoin croissant en logements. Ce complexe se trouve lui aussi en périphérie, à proximité de la B 80 ; il présente pour seul avantage la proximité d'un parc. Son paysage est marqué par de longs alignements de bâtiments, et la structure des voies de circulation a été mal conçue.

L'analyse du devenir de chaque complexe montre la complexité des facteurs dans l'attractivité d'un lieu. Ne compte pas seulement dans la planification des transformations du quartier le niveau de réhabilitation du bâti ou la qualité des services, mais aussi la position du complexe par rapport à l'ensemble urbain. Certains complexes peuvent cumuler les handicaps, le complexe VI en étant le meilleur exemple, d'autres au contraires, bien que présentant certaines lacunes, peuvent tirer profit de leur situation : le complexe VIII, dont les immeubles présentent un faible taux de réhabilitation, demeure relativement attractif grâce à la proximité du campus universitaire, du centre de Halle, et des rives de la Saale. 


\section{Conclusion}

Une ville mono-industrielle comme Eisenhüttenstadt, entièrement élaborée par le pouvoir socialiste, connaît aujourd'hui le sort difficile des petits pôles industriels isolés ; elle a été fortement touchée par la désindustrialisation de l'après-réunification. Son tissu urbain est majoritairement composé de grands ensembles. Certains quartiers font l'objet de mesures de réhabilitation; un complexe d'habitation va disparaître presque en totalité. Il s'agit d'un quartier datant des années quatre-vingt, qui présentait de très grandes lacunes au niveau de la qualité du bâti et du paysage. Le cas de Halle-Neustadt se pose de manière quelque peu différente, bien qu'elle ait été à l'origine également une ville modèle du socialisme. Aujourd'hui, la question de son devenir s'intègre dans une réflexion générale sur la ville de Halle/Saale. Aux réflexions générales sur le maintien de la structure et du paysage de la ville socialiste, se sont ajoutées les difficultés techniques et les nécessaires recherches de consensus entre les multiples acteurs. On s'oriente dans le cas de Halle-Neustadt vers le maintien de son caractère urbain et de son identité, ce qui n'est pas le cas pour un autre quartier de grands ensembles de la ville (Silberhöhe) pour lequel a été retenue l'option de la villeforêt (Waldstadt).

51 Le devenir des quartiers et villes de la période socialiste se pose de manière aiguë dans un contexte de fort déclin démographique, d'autant que l'on s'oriente plutôt vers une mise en valeur des quartiers anciens.

\section{BIBLIOGRAPHIE}

BMVBS (Bundesministeriums für Verkehr, Bau und Stadtentwicklung), BBR (Bundesamtes für Bauwesen und Raumordnung) (2006). - Stadtumbau Ost, Stand und Perspektiven, Berlin, 89 p.

BMVBW (Bundesministerium für Verkehr, Bau- und Wohnungswesen) (2002). - Programm Stadtumbau Ost, Für lebenswerte Städte und attraktives Wohnen; Merkblatt über die Finanzhilfen des Bundes, Berlin, $17 \mathrm{p}$. 
BMVBW (Bundesministerium für Verkehr, Bau- und Wohnungswesen), BBR (Bundesamtes für Bauwesen und Raumordnung) (2001). - Stadtumbau in den neuen Ländern, Berlin, 103 p.

COUDROY DE LILLE L. (2004). - « Une idéologie du préfabriqué ? ». In : DUFAUX F., FOURCAUT A. - dir., Le monde des grands ensembles, Paris, Créaphis, p. 90-95.

Fachbereich Stadtentwicklung und- planung, Ressort Stadtplanung (2001). -Neuordnungskonzepte für die Stadtteile Silberhöhe und Halle-Neustadt in Halle (Saale); Kurzfassung, Halle, 11 p.

Fachbereich Stadtentwicklung und -planung, Ressort Stadtplanung (2006). - Stadtumbaugebiet Halle-Neustadt : Stadtumbaukonzept, Fortschreibung des Neuordnungskonzeptes aus dem Jahr 2001, Halle, $79 \mathrm{p}$.

HANNEMANN C. (2003). - «Schrumpfende Städte in Ostdeutschland, Ursachen und Folgen einer Stadtentwicklung ohne Wirtschaftswachstum », www.bpb.de/publikationen/RGEU7W.html, 14 novembre 2006.

HOLZ J.-M. (1997). - « Recompositions en Allemagne ». In : Maurel M.-C., Recomposition de l'Europe médiane, Paris, SEDES, coll. « Dossiers des Images Economiques du Monde », p. 157-188.

Kommission « Wohnungswirtschaftlicher Strukturwandel in den neuen Bundesländern » (2000).

- Bericht, Kurzfassung, 11 p.

корР А. (1978). - L'architecture de la période stalinienne, Grenoble, P.U. de Grenoble, 414 p.

корР A. (1972). - Ville et révolution, Paris, Seuil, coll. « Points civilisation », nº 32.

LEVY J., LUSSAUlT M. - dir. (2003). - Dictionnaire de la géographie, Paris, Belin, 1033 p.

LINDEN P. (2004) - «Stalinstadt à l'heure du déclin », Le monde Diplomatique, août 2004, www.monde-diplomatique.fr/2004/08/LINDEN/11499, 14 novembre 2006

MERLIN P., CHOAY F. - dir. (2005). - Dictionnaire de l'urbanisme et de l'aménagement, Paris, P.U.F., $3^{\mathrm{e}}$ édition, coll. «Quadrige-Dicos poche », $992 \mathrm{p}$.

MÜLLER E. (1997). - « Vorwort », Beiträge zur regionalen Geographie, 45, p. 5.

POHL R. (2003). - «Wirtschaftsstandort Saxe-Anhalt. Stadtumbau als Chance ?», www.ak-lsa.de/ download/03-neujahrsempfang-gedanken.pdf, 22 juin 2006.

Rowell J. (2004). - « Du grand ensemble au « complexe d'habitation socialiste » ». In : DUFAUX F., FOURCAUT A. - dir., Le monde des grands ensembles, Paris, Créaphis, p. 97-109.

\section{NOTES}

1. Source : Institut für Arbeitsmarkt- und Berufsforschung der Bundesagentur für Arbeit, www.iab.de, 15 octobre 2005

2. Constituée en 2000 à l'instigation du Ministre fédéral des transports, de la construction et du logement, du délégué du gouvernement aux nouveaux Länder et du ministre d'Etat à la Chancellerie.

3. L'expression "concept intégré de développement urbain» a été utilisée au moment du concours ; à présent, on emploie plutôt (mais non exclusivement) celle de « concept urbanistique de développement".

4. Source : Dossier ville : France-Allemagne : une confrontation intéressante sur le site internet : www.gouv.fr/infos/dossiers/france-allemagne.html,15 avril 2006. 
5. Source : www. sozialestadt.de/français/veroeffentlichungen/zwischenbilanz/1zwischenbilanz.shtml,20 mars 2006.

\section{RÉSUMÉS}

Les difficultés que connaissent aujourd'hui les nouveaux Länder se traduisent notamment par un déclin des villes dont la gravité a conduit le gouvernement fédéral à mettre en place un programme d'aides, le programme Stadtumbau Ost (Rénovation Urbaine à l'est). Si les quartiers anciens ont été les premiers concernés par les départs massifs, une évolution récente fait des quartiers de grands ensembles des zones de plus en plus fragiles. Les logements vides nombreux, la sous-utilisation des équipements collectifs, la dégradation de certains immeubles sont quelques unes des conséquences de ces départs. Le programme Stadtumbau Ost incite les villes à mettre en place des concepts de développement urbain, afin d'organiser les opérations de démolition et de réhabilitation qui prennent ici une ampleur exceptionnelle. Dans le cas d'Eisenhüttenstadt, ville nouvelle créée autour d'un complexe sidérurgique, on a choisi la démolition totale d'un quartier. Halle-Neustadt, qui a également été une ville nouvelle socialiste avant de devenir un des grands ensembles de Halle/Saale, illustre les difficultés à mettre en place une stratégie urbaine dans ce grand ensemble de la taille d'une ville moyenne.

Today, the difficulties confronting the new Länder are expressed notably in declining towns, a problem which has lead the Federal government to design a support programme, Stadtumbau Ost (Urban Restructuring East). While old residential areas were the first to be affected by falling population, recent changes have made large scale housing developments increasingly dilapidated. Numerous empty dwelling units, under-used public infrastructure and decay of council flats are some of the consequences of demographic shrinkage. The programme Stadtumbau Ost encourages towns to establish guiding principles of urban development in order to enact the changes required by new circumstances. The transformation involves demolition, rehabilitation and changes to public infrastructure. In the case of Eisenhüttenstadt, a new town created in the vicinity of a steel factory, a whole district has been demolished. Another example, Halle-Neustadt, which was also a new socialist town before becoming one of Halle/Saale's large scale housing developments, illustrates the difficulties involved in establishing an urban strategy in this large housing development as big as a medium-sized town.

Die aktuellen Probleme der neuen Länder finden ihren Ausdruck in der Schrumpfung der Städte, dessen Ausmaß die Bundesregierung dazu veranlasst hat, das Förderprogramm Stadtumbau Ost aufzulegen. Waren zunächst die Altbaubestände am stärksten von hohen Abwanderungen betroffen, so stellen in jüngerer Zeit die Großwohnsiedlungen die größten Problemgebiete dar. Hohe Leerstände, unterausgelastete Gemeinschaftseinrichtungen, der Verfall vieler Gebäude sind nur einige Auswirkungen der starken Abwanderung. Das Programm Stadtumbau Ost unterstützt die Städte und Gemeinden, Stadtentwicklungskonzepte zu erstellen, in deren Rahmen Rückbau(Abriß) und Sanierungsmaßnahmen erheblichen Ausmaßes umgesetzt werden. Im Falle von Eisenhüttenstadt, einer neuen um ein Eisenhüttenkombinat gegründeten Stadt, hat man sich für den Totalabriss eines ganzen Stadtviertels entschieden. Am Beispiel von Halle-Neustadt, ebenfalls eine neue sozialistische Stadt, bevor diese eine der Großwohnsiedlungen von Halle 
(Saale) wurde, wird deutlich, wie schwer es ist, eine Stadtentwicklungsstrategie für eine Großwohnsiedlung von der Größe einer Mittelstadt zu entwickeln und umzusetzen.

\section{INDEX}

Schlüsselwörter : Großwohnsiedlungen, Neue Länder, Stadtentwicklungskonzept, Stadtumbau Mots-clés : concept intégré de développement urbain, Grands ensembles, nouveaux Länder, rénovation urbaine

Keywords : guiding principle of urban development, Large scale housing developments, new Länder, urban restructuring

\section{AUTEUR}

\section{AXELLE DE GASPÉRIN}

Doctorante - CERPA - Université de Nancy 2 - 23, boulevard Albert $1^{\text {er }}-54015$ Nancy CEDEX 